\title{
A SEPARATOR THEOREM FOR NONPLANAR GRAPHS
}

\author{
NOGA ALON, PAUL SEYMOUR, AND ROBIN THOMAS
}

\section{INTRODUCTION}

A separation of a graph $G$ is a pair $(A, B)$ of subsets of $V(G)$ with $A \cup B=$ $V(G)$, such that no edge of $G$ joins a vertex in $A-B$ to a vertex in $B-A$. Its order is $|A \cap B|$. A well-known theorem of Lipton and Tarjan [2] asserts the following. ( $\mathbf{R}^{+}$denotes the set of nonnegative real numbers. If $w: V(G) \rightarrow \mathbf{R}^{+}$ is a function and $X \subseteq V(G)$, we denote $\sum(w(v): v \in X)$ by $w(X)$.)

(1.1) Let $G$ be a planar graph with $n$ vertices, and let $w: V(G) \rightarrow \mathbf{R}^{+}$be a function. Then there is a separation $(A, B)$ of $G$ of order $\leq 2 \sqrt{2} \sqrt{n}$, such that $w(A-B), w(B-A) \leq \frac{2}{3} w(V(G))$.

Our object is to prove an extension of (1.1) for nonplanar graphs with a fixed excluded "minor." A graph $H$ is a minor of a graph $G$ if $H$ can be obtained from a subgraph of $G$ by contracting edges. By an $H$-minor of $G$ we mean a minor of $G$ isomorphic to $H$. Thus, the Kuratowski-Wagner theorem asserts that planar graphs are those without $K_{5}$ - or $K_{3,3}$-minors. We prove the following:

(1.2) Let $h \geq 1$ be an integer, let $G$ be a graph with $n$ vertices and with no $K_{h}$-minor, and let $w: V(G) \rightarrow \mathbf{R}^{+}$be a function. Then there is a separation $(A, B)$ of $G$ of order $\leq h^{3 / 2} n^{1 / 2}$ such that $w(A-B), w(B-A) \leq \frac{2}{3} w(V(G))$.

Our thanks to N. Linial, who pointed out several years ago to the second author that a result like (1.2) was probably true. We think that the expression $h^{3 / 2} n^{1 / 2}$ in (1.2) is not the best possible, and that $O\left(h n^{1 / 2}\right)$ is the correct answer, but have not been able to decide this. If true, this would generalize a result of Gilbert, Hutchinson, and Tarjan [1] that every graph with $n$ vertices and genus $g$ has a "separator" of order $\leq O\left(g^{1 / 2} n^{1 / 2}\right)$, because $K_{h}$ has genus $\geq \Omega\left(h^{2}\right)$. Every 3-regular expander with $n$ vertices is a graph with no $K_{h}$-minor for $h=c n^{1 / 2}$, and with no separator of size $d n$, for appropriately chosen positive constants $c$ and $d$; and hence the estimate $O\left(h n^{1 / 2}\right)$ would be the best possible.

Received by the editors December 11, 1989 and, in revised form, March 1990; the contents of this paper were presented at the 22nd STOC conference, Baltimore, Maryland, 1990.

1980 Mathematics Subject Classification (1985 Revision). Primary 05C40.

The first author's research was carried out under a consulting agreement with Bellcore. 
We observe also that since $K_{h}$ contains an $H$-minor for every simple graph $H$ with $h$ vertices, (1.2) is equivalent to the following.

(1.3) Let $h \geq 1$ be an integer, let $G$ be a graph with $n$ vertices and with no $H$-minor, where $H$ is an arbitrary simple graph with $h$ vertices, and let $w: V(G) \rightarrow \mathbf{R}^{+}$be a function. Then there is a separation $(A, B)$ of $G$ of order $\leq h^{3 / 2} n^{1 / 2}$ such that $w(A-B), w(B-A) \leq \frac{2}{3} w(V(G))$.

If $G$ is a graph and $X \subseteq V(G)$, an $X$-flap is the vertex set of some component of $G \backslash X$ (the graph obtained from $G$ by deleting $X$ ). Let $w: V(G) \rightarrow \mathbf{R}^{+}$ be a function. If $X \subseteq V(G)$ is such that $w(F) \leq \frac{2}{3} w(V(G))$ for every $X$-flap $F$ then it is easy to find a separation $(A, B)$ with $A \cap B=X$ such that $w(A-B), w(B-A) \leq \frac{2}{3} w(V(G))$. (If $w(F) \geq \frac{1}{3} w(V(G))$ for some $X$-flap $F$, take the separation $(F \cup X, V(G)-F)$. If not, let the $X$-flaps be $F_{1}, \ldots, F_{k}$ and choose $j$ with $1 \leq j \leq k$ maximal such that $\sum_{1 \leq i \leq j} w\left(F_{i}\right) \leq \frac{2}{3} w(V(G))$; and take the separation $(H \cup X, V(G)-H)$, where $H=\bigcup_{1 \leq i \leq j} F_{i}$.) Thus, (1.2) is implied by the following:

(1.4) Let $h \geq 1$ be an integer, let $G$ be a graph with $n$ vertices and with no $K_{h}$-minor, and let $w: V(G) \rightarrow \mathbf{R}^{+}$be a function. Then there exists $X \subseteq V(G)$ with $|X| \leq h^{3 / 2} n^{1 / 2}$ such that $w(F) \leq \frac{1}{2} w(V(G))$ for every $X$-flap $F$.

Lipton and Tarjan [2] gave an algorithm to find a separation $(A, B)$ as in (1.1) in linear time. We have not been able to do as well, but we shall show the following:

(1.5) There is an algorithm with running time $O\left(h^{1 / 2} n^{1 / 2} m\right)$, which takes as input an integer $h \geq 1$, a graph $G$ (where $n=|V(G)|$ and $m=|V(G)|+$ $|E(G)|)$, and a function $w: V(G) \rightarrow \mathbf{R}^{+}$. It outputs either

(a) a $K_{h}$-minor of $G$, or

(b) a subset $X \subseteq V(G)$ with $|X| \leq h^{3 / 2} n^{1 / 2}$ such that $w(F) \leq \frac{1}{2} w(V(G))$ for every $X$-flap $F$.

Several algorithmic applications of this result appear in [4]

By a haven of order $k$ in $G$ we mean a function $\beta$ which assigns to each subset $X \subseteq V(G)$ with $|X| \leq k$ an $X$-flap $\beta(X)$, in such a way that if $X \subseteq Y$ and $|Y| \leq k$ then $\beta(Y) \subseteq \beta(X)$. Now if (1.4) is false, then for each $X \subseteq V(G)$ with $|X| \leq h^{3 / 2} n^{1 / 2}$ there is a unique $X$-flap, say $\beta(X)$, with $w(\beta(X))>$ $\frac{1}{2} w(V(G))$; and $\beta$ thus defined is evidently a haven of order $h^{3 / 2} n^{1 / 2}$. Thus (1.4) is implied by the following:

(1.6) Let $h \geq 1$ be an integer and let $G$ be a graph with $n$ vertices with a haven of order $h^{3 / 2} n^{1 / 2}$. Then $G$ has a $K_{h}$-minor.

While (1.6) is more compact and more general than (1.4), it seems difficult to formulate a corresponding generalization of (1.5). We shall content ourselves, therefore, with proving (1.5) and (1.6) separately. 
Let us mention an application of (1.6). A tree-decomposition of a graph $G$ is a pair $(T, W)$, where $T$ is a tree and $W=\left(W_{t}: t \in V(T)\right)$ is a family of subsets of $V(G)$, such that

(i) $\bigcup\left(W_{t}: t \in V(T)\right)=V(G)$, and for every $e \in E(G)$ there exists $t \in$ $V(T)$ such that $W_{t}$ contains both ends of $e$;

(ii) if $t_{1}, t_{2}, t_{3} \in V(T)$ and $t_{2}$ lies on the path between $t_{1}$ and $t_{3}$ then $W_{t_{1}} \cap W_{t_{3}} \subseteq W_{t_{2}}$.

The tree-width of $G$ is the minimum $k$ such that there is a tree-decomposition $(T, W)$ of $T$ satisfying $\left|W_{t}\right| \leq k+1$ for all $t \in V(T)$. The following is proved in [3]:

(1.7) If $k \geq 0$ is an integer then $G$ has a haven of order $\geq k$ if and only if the tree-width of $G$ is at least $k$.

From (1.6) and (1.7) we deduce

(1.8) Let $h \geq 1$ be an integer and let $G$ be a graph with $n$ vertices and with tree-width at least $h^{3 / 2} n^{1 / 2}$. Then $G$ has a $K_{h}$-minor.

\section{FINDING SMALL CONNECTING TREES}

We shall need the following lemma:

(2.1) Let $G$ be a graph with $n$ vertices, let $A_{1}, \ldots, A_{k} \subseteq V(G)$, and let $r \in \mathbf{R}^{+}$with $r \geq 1$. Then either

(i) there is a tree $T$ in $G$ with $|V(T)| \leq r$ such that $V(T) \cap A_{i} \neq \varnothing$ for $i=1, \ldots, k$, or

(ii) there exists $Z \subseteq V(G)$ with $|Z| \leq(k-1) n / r$, such that no $Z$-flap intersects all of $A_{1}, \ldots, A_{k}$.

Proof. We may assume that $k \geq 2$. Let $G^{1}, \ldots, G^{k-1}$ be isomorphic copies of $G$, mutually disjoint. For each $v \in V(G)$ and $1 \leq i \leq k-1$, let $v^{i}$ be the corresponding vertex of $G^{i}$. Let $J$ be the graph obtained from $G^{1} \cup \cdots \cup G^{k-1}$ by adding, for $2 \leq i \leq k-1$ and all $v \in A_{i}$, an edge joining $v^{i-1}$ and $v^{i}$. Let $X=\left\{v^{1}: v \in A_{1}\right\}$ and $Y=\left\{v^{k-1}: v \in A_{k}\right\}$. For each $u \in V(J)$, let $d(u)$ be the number of vertices in the shortest path of $J$ between $X$ and $u$ (or $\infty$ if there is no such path). There are two cases:

Case 1. $d(u) \leq r$ for some $u \in Y$.

Let $P$ be a path of $J$ between $X$ and $Y$ with $\leq r$ vertices. Let

$$
S=\left\{v \in V(G): v^{i} \in V(P) \text { for some } i, 1 \leq i \leq k-1\right\} .
$$

Then $|S| \leq|V(P)| \leq r$, the subgraph of $G$ induced on $S$ is connected, and $\left|S \cap A_{i}\right| \neq \varnothing$ for $1 \leq i \leq k$. Thus (i) holds.

Case 2. $d(u)>r$ for all $u \in Y$. 
Let $t$ be the least integer with $t \geq r$. For $1 \leq j \leq t$, let $Z_{j}=\{u \in$ $V(J): d(u)=j\}$. Since $|V(J)|=(k-1) n$ and $Z_{1}, \ldots, Z_{t}$ are mutually disjoint, one of them, say $Z_{j}$, has cardinality $\leq(k-1) n / t \leq(k-1) n / r$. Now every path of $J$ between $X$ and $Y$ has a vertex in $Z_{j}$, because $d(u) \geq j$ for all $u \in Y$. Let

$$
Z=\left\{v \in V(G): v^{i} \in Z_{j} \text { for some } i, 1 \leq i \leq k-1\right\} \text {. }
$$

Then $|Z| \leq\left|Z_{j}\right| \leq(k-1) n / r$, and we claim that $Z$ satisfies (ii). Suppose that $F$ is a $Z$-flap of $G$ which intersects all of $A_{1}, \ldots, A_{k}$. Let $a_{i} \in F \cap A_{i}$ $(1 \leq i \leq k)$, and for $1 \leq i \leq k-1$ let $P_{i}$ be a path of $G$ with $V\left(P_{i}\right) \subseteq F$ and with ends $a_{i}, a_{i+1}$. Let $P^{i}$ be the path of $G^{i}$ corresponding to $P_{i}$. Then $V\left(P^{1}\right) \cup \cdots \cup V\left(P^{k-1}\right)$ includes the vertex set of a path of $J$ between $X$ and $Y$, and yet is disjoint from $Z_{j}$, a contradiction. Thus, there is no such $F$, and so (ii) holds.

We observe that the proof of (2.1) is easily converted to an algorithm with running time $O(\mathrm{~km})$, which, with input $G, r$, and $A_{1}, \ldots, A_{k}$ as in (2.1) (where $m=|V(G)|+|E(G)|$ ), computes either a tree $T$ as in (i) or a set $Z$ as in (ii).

It would be desirable to replace the expression $(k-1) n / r$ in (ii) by some $f(k) n / r$, where $f(k)=o(k)$, because there would be a corresponding improvement in the expression $h^{3 / 2} n^{1 / 2}$ of (1.2). We do not know if this is possible, but we suspect not. Indeed, let $G$ be the "cube" with vertex set

$$
\left\{\left(x_{1}, \ldots, x_{d}\right): x_{1}, x_{2}, \ldots, x_{d} \in\{0,1\}\right\},
$$

where $\left(x_{1}, \ldots, x_{d}\right)$ and $\left(x_{1}^{\prime}, \ldots, x_{d}^{\prime}\right)$ are adjacent if $\left|x_{1}-x_{1}^{\prime}\right|+\cdots+\left|x_{d}-x_{d}^{\prime}\right|=$ 1. For $1 \leq i \leq d$, let

$$
A_{i}=\left\{\left(x_{1}, \ldots, x_{d}\right): x_{i}=0\right\}, \quad A_{d+i}=\left\{\left(x_{1}, \ldots, x_{d}\right): x_{i}=1\right\},
$$

and let $k=2 d$. Then certainly every tree in $G$ which meets all of $A_{1}, \ldots, A_{k}$ has at least $d+1$ vertices, and yet we suspect that, for any $Z \subseteq V(G)$ with $|Z|<2^{d-1}$, some $Z$-flap intersects all of $A_{1}, \ldots, A_{k}$. If so, this would show that (2.1) is best possible up to a constant factor.

\section{Proof OF THE THEOREM}

First we prove (1.6), and then adapt the proof to yield an algorithm for (1.5). Let $G$ be a graph. By a covey in $G$ we mean a set $\mathscr{C}$ of (nonnull) trees of $G$, mutually vertex-disjoint, such that for all distinct $C_{1}, C_{2} \in \mathscr{C}$ there is an edge of $G$ with one end in $V\left(C_{1}\right)$ and the other in $V\left(C_{2}\right)$. Thus, if $G$ has a covey of cardinality $h$ then it has a $K_{h}$-minor.

Proof of (1.6). Let $\beta$ be a haven in $G$ of order $h^{3 / 2} n^{1 / 2}$. Choose $X \subseteq V(G)$ and a covey $\mathscr{C}$ with $|\mathscr{C}| \leq h$ such that

(i) $X \subseteq \cup(V(C): C \in \mathscr{C})$,

(ii) $|X \cap V(C)| \leq h^{1 / 2} n^{1 / 2}$ for each $C \in \mathscr{C}$, 
(iii) $V(C) \cap \beta(X)=\varnothing$ for each $C \in \mathscr{C}$, and

(iv) subject to (i), (ii), and (iii), $|\mathscr{C}|+|X|+3|\beta(X)|$ is minimum.

(This is certainly possible; setting $\mathscr{C}=X=\varnothing$ satisfies (i), (ii), and (iii).) Let $\mathscr{C}=\left\{C_{1}, \ldots, C_{k}\right\}$. We suppose for a contradiction that $k<h$. For $1 \leq i \leq k$, let $A_{i}$ be the set of all $v \in \beta(X)$ adjacent in $G$ to a vertex of $C_{i}$. Let $G^{\prime}$ be the restriction of $G$ to $\beta(X)$. By (2.1) applied to $G^{\prime}$ with $r=h^{1 / 2} n^{1 / 2}$, one of the following cases holds:

Case 1. There is a tree $T$ of $G^{\prime}$ with $|V(T)| \leq h^{1 / 2} n^{1 / 2}$, such that $V(T) \cap A_{i} \neq$ $\varnothing$ for $1 \leq i \leq k$. Let $\mathscr{C}^{\prime}=\mathscr{C} \cup\{T\}$ and $X^{\prime}=X \cup V(T)$; then $\mathscr{C}^{\prime}$ is a covey and for each $C \in \mathscr{C}^{\prime}$,

$$
V(C) \cap \beta\left(X^{\prime}\right) \subseteq V(C) \cap(\beta(X)-V(T))=\varnothing .
$$

This contradicts (iv).

Case 2. There exists $Z \subseteq \beta(X)$ with $|Z| \leq(k-1)|\beta(X)| / h^{1 / 2} n^{1 / 2} \leq h^{1 / 2} n^{1 / 2}$ such that no $Z$-flap of $G^{\prime}$ intersects all of $A_{1}, \ldots, A_{k}$. Let $Y=X \cup Z$. Since $k \leq h-1$, it follows that $|Y| \leq h^{3 / 2} n^{1 / 2}$, and so $\beta(Y)$ exists and $\beta(Y) \subseteq \beta(X)$. Since $\beta(Y)$ is a $Z$-flap of $G^{\prime}$ there exists $i$ with $1 \leq i \leq k$ such that $\beta(Y) \cap A_{i}=\varnothing$. Extend $C_{i}$ to a maximal tree $C_{i}^{\prime}$ of $G$ disjoint from $\beta(Y)$ and from each $C_{j}(j \neq i)$. Let $Z^{\prime}=V\left(C_{i}^{\prime}\right) \cap Z$, let $X^{\prime}=Z^{\prime} \cup\left(X-V\left(C_{i}\right)\right)$, and let $W=V\left(C_{i}^{\prime}\right) \cup(V(G)-\beta(X))$.

We claim that $\beta\left(X^{\prime}\right) \cap W=\varnothing$. For suppose not. Since $\beta(Y) \subseteq \beta\left(X^{\prime}\right)$, there is a path of $G$ between $W$ and $\beta(Y)$ contained within $\beta\left(X^{\prime}\right)$ and hence disjoint from $X^{\prime}$. Since $W \cap \beta(Y)=\varnothing$, there are two consecutive vertices $u, v$ of this path with $u \in W$ and $\nu \in V(G)-W \subseteq \beta(X)$. Since $u, v$ are adjacent it follows that $u \in X \cup \beta(X)$, and so

$$
u \in(X \cup \beta(X)) \cap\left(W-X^{\prime}\right) \subseteq V\left(C_{i}^{\prime}\right) .
$$

Since $v \notin W$ it follows from the maximality of $C_{i}^{\prime}$ that $v \in \beta(Y)$. Since $u \notin \beta(Y)$ we deduce that $u \in Y$, and so

$$
u \in Y \cap\left(V\left(C_{i}^{\prime}\right)-X^{\prime}\right) \subseteq V\left(C_{i}\right)
$$

But then $v \in A_{i}$, which is impossible since $A_{i} \cap \beta(Y)=\varnothing$. This proves our claim that $\beta\left(X^{\prime}\right) \cap W=\varnothing$. Hence, $\beta\left(X^{\prime}\right) \subseteq \beta(X)$. Let $\mathscr{C}^{\prime}=\left(\mathscr{C}-\left\{C_{i}\right\}\right) \cup\left\{C_{i}^{\prime}\right\}$; then $\mathscr{C}^{\prime}$ is a covey. We observe that

(i) $X^{\prime} \subseteq \bigcup\left(V(C): C \in \mathscr{C}^{\prime}\right)$; for $Z^{\prime} \subseteq V\left(C_{i}^{\prime}\right)$,

(ii) $\left|X^{\prime} \cap V(C)\right| \leq h^{1 / 2} n^{1 / 2}$ for each $C \in \mathscr{C}^{\prime}$; for if $C \neq C_{i}^{\prime}$ then $X^{\prime} \cap$ $V(C)=X \cap V(C)$, and $X^{\prime} \cap V\left(C_{i}^{\prime}\right)=Z^{\prime}$, and

(iii) $V(C) \cap \beta\left(X^{\prime}\right)=\varnothing$ for each $C=\mathscr{C}^{\prime}$; for $\beta\left(X^{\prime}\right) \cap W=\varnothing$, as we have seen. 
By (iv),

$$
\left|\mathscr{C}^{\prime}\right|+\left|X^{\prime}\right|+3\left|\beta\left(X^{\prime}\right)\right| \geq|\mathscr{C}|+|X|+3|\beta(X)| .
$$

But $\left|\mathscr{C}^{\prime}\right|=|\mathscr{C}|$ and $X^{\prime} \cup \beta\left(X^{\prime}\right) \subseteq(X \cup \beta(X))-\left(X \cap V\left(C_{i}\right)\right)$, and so $X \cap V\left(C_{i}\right)=$ $\varnothing$. Then $\mathscr{C}-\left\{C_{i}\right\}, X$ satisfy (i), (ii), and (iii), contrary to (iv).

In both cases, therefore, we have obtained a contradiction. Thus our assumption that $k<h$ was incorrect, and so $k=h$ and $G$ has a $K_{h}$-minor, as required.

Now let us convert the proof of (1.6) to an algorithm for (1.5). The main difference will be that we shall keep the sets $X \cap V(C)$ as large as possible, to improve the running time. Let $h, G, w$ be the input, and let $r=\left\lfloor h^{1 / 2} n^{1 / 2}\right\rfloor$. Set $X_{0}=\mathscr{C}_{0}=\varnothing$ and $B_{0}=V(G)$, and begin the first iteration. In general, at the beginning of the $t$ th iteration, we have a subset $X_{t-1} \subseteq V(G)$, a covey $\mathscr{C}_{t-1}$ with $\left|\mathscr{C}_{t-1}\right| \leq h$, and a subset $B_{t-1} \subseteq V(G)$ which is a union of $X_{t-1}$-flaps, such that

(i) $X_{t-1} \subseteq \bigcup\left(V(C): C \in \mathscr{C}_{t-1}\right)$,

(ii) $\left|X_{t-1} \cap V(C)\right|=r$ for each $C \in \mathscr{C}_{t-1}$,

(iii) $V(C) \cap B_{t-1}=\varnothing$ for each $C \in \mathscr{C}_{t-1}$,

(iv) $w(F) \leq \frac{1}{2} w(V(G))$ for each $X_{t-1}$-flap $F$ with $F \nsubseteq B_{t-1}$.

(1) Let $\left|\mathscr{C}_{t-1}\right|=k$. If $k=h$ we have found a $K_{h}$-minor; we output (a) and stop. Otherwise we go to step (2).

(2) We compute all the $X_{t-1}$-flaps included in $B_{t-1}$. If $w(F) \leq \frac{1}{2} w(V(G))$ for every such $X_{t-1}$-flap $F$, we output (b) (with $X=X_{t-1}$ ) and stop. Otherwise, let $F$ be the unique $X_{t-1}$-flap with $w(F)>\frac{1}{2} w(V(G))$. If $|F|<h^{1 / 2} n^{1 / 2}$ we output (b) (with $X=X_{t-1} \cup F$ ) and stop. Otherwise we go to step (3).

(3) Let $\mathscr{C}_{t-1}=\left\{C_{1}, \ldots, C_{k}\right\}$. For $1 \leq i \leq k$, let $A_{i}$ be the set of all $v \in F$ with a neighbor in $V\left(C_{i}\right)$. If $A_{i}=\varnothing$ for some $i$, we set $X_{t}=$ $X_{t-1}-V\left(C_{i}\right), \mathscr{C}_{t}=\mathscr{C}_{t-1}-\left\{C_{i}\right\}, B_{t}=F$, and return to step (1) for the next iteration. Otherwise we go to step (4).

(4) Let $G^{\prime}$ be the restriction of $G$ to $F$. We apply (2.1) to $G^{\prime}$ and $A_{1}, \ldots, A_{k}$. We obtain either:

(i) a tree $T$ of $G^{\prime}$ with $|V(T)| \leq r$ such that $V(T) \cap A_{i} \neq \varnothing$ for each $i$, or

(ii) a subset $Z \subseteq V\left(G^{\prime}\right)$ with $|Z| \leq(k-1) n / r \leq r$ such that no $Z$-flap of $G^{\prime}$ intersects all of $A_{1}, \ldots, A_{k}$.

In the first case we go to step (5), and in the second to step (6).

(5) Given $T$ as in (4)(i), we enlarge $T$ to a tree $T^{\prime}$ of $G^{\prime}$ with $\left|V\left(T^{\prime}\right)\right|=r$ (this is possible since $|F| \geq r$ ). We set $X_{t}=X_{t-1} \cup V\left(T^{\prime}\right), \mathscr{C}_{t}=\mathscr{C}_{t-1} \cup\left\{T^{\prime}\right\}$, $B_{t}=F-V\left(T^{\prime}\right)$, and return to step (1) for the next iteration.

(6) Given $Z$ as in (4)(ii), let $Y=X_{t-1} \cup Z$. If $w(D) \leq \frac{1}{2} w(V(D))$ for every $Y$-flap $D$ of $G$, we output (b) (with $X=Y$ ) and stop. Otherwise, let $D$ be the unique $Y$-flap with $w(D)>\frac{1}{2} w(V(G))$. Now $D$ is a $Z$-flap of $G^{\prime}$, 
and so we may choose $i$ with $1 \leq i \leq k$ such that $D \cap A_{i}=\varnothing$. Extend $C_{i}$ to a maximal tree $C_{i}^{\prime}$ of $G$ disjoint from $D$ and from each $C_{j}(j \neq i)$. Since $A_{i} \cap \beta(Y)=\varnothing$, it follows that $A_{i} \subseteq V\left(C_{i}^{\prime}\right)$. Let

$$
X^{\prime}=\left(V\left(C_{i}^{\prime}\right) \cap Z\right) \cup\left(X_{t-1}-V\left(C_{i}\right)\right) ;
$$

then, as in the proof of (1.6), it follows that

$$
F^{\prime} \cap\left(V\left(C_{i}^{\prime}\right) \cup \bigcup\left(V\left(C_{j}\right): 1 \leq j \leq k, j \neq i\right)\right)=\varnothing
$$

for any $X^{\prime}$-flap $F^{\prime}$ of $G$ with $w\left(F^{\prime}\right)>\frac{1}{2} w(V(G))$. Extend $C_{i}^{\prime}$ to a maximal tree $T$ of $G$ disjoint from each $C_{j}(j \neq i)$ and with $|V(T) \cap(Z \cup D)| \leq r$. Since $\varnothing \neq A_{i} \subseteq V\left(C_{i}^{\prime}\right)$ and $|F| \geq r$, it follows that $|V(T) \cap F| \geq r$, and so we may choose $Z^{\prime}$ with $\left|Z^{\prime}\right|=r$ such that

$$
V(T) \cap(Z \cup D) \subseteq Z^{\prime} \subseteq V(T) \cap F .
$$

We set $X_{t}=\left(X_{t-1}-V\left(C_{i}\right)\right) \cup Z^{\prime}, \mathscr{C}_{t}=\left(\mathscr{C}_{t-1}-\left\{C_{i}\right\}\right) \cup\{T\}, B_{t}=D-Z^{\prime}$, and return to (1) for the next iteration. (We observe that any $X_{t}$-flap $F^{\prime}$ of $G$ with $w\left(F^{\prime}\right)>\frac{1}{2} w(V(G))$ is a subset of an $X^{\prime}$-flap with the same property, since $X^{\prime} \subseteq X_{t}$, and hence is disjoint from each member of $\mathscr{C}_{t}$ and is a subset of $B_{t}$.)

This completes the description of the algorithm (apart from the simple data structures used, which we omit) and the proof of correctness. To analyze the running time, we observe that the most time-consuming part of each iteration is step (4), which takes time $\leq O(\mathrm{hm})$. Since $\left|X_{0}\right|+2\left|B_{0}\right|=2 n$ and in each iteration the quantity $\left|X_{t}\right|+2\left|B_{t}\right|$ is reduced by at least $r$, there are at most $2 n / r \leq O\left(h^{-1 / 2} n^{1 / 2}\right)$ iterations, and so the total running time is at most $O\left(h^{1 / 2} n^{1 / 2} m\right)$, as claimed.

It may be that by using more sophisticated, dynamic data structures, the algorithm can be implemented more efficiently, but at the moment we do not see how to do so.

\section{REFERENCES}

1. J. R. Gilbert, J. P. Hutchinson, and R. E. Tarjan, A separation theorem for graphs of bounded genus, J. Algorithms 5 (1984), 391-407.

2. R. J. Lipton and R. E. Tarjan, A separator theorem for planar graphs, SIAM J. Appl. Math. 36 (1979), 177-189.

3. P. D. Seymour and R. Thomas, Graph searching, and a minimax theorem for tree-width, J. Combin. Theory, Ser. B (to appear).

4. N. Alon, P. D. Seymour, and R. Thomas, A separator theorem for graphs with an excluded minor and its applications (Proc. 22nd STOC, Baltimore, Maryland, 1990), ACM Press, 293-299. 
ABSTRACT. Let $G$ be an $n$-vertex graph with no minor isomorphic to an $h$ vertex complete graph. We prove that the vertices of $G$ can be partitioned into three sets $A, B, C$ such that no edge joins a vertex in $A$ with a vertex in $B$, neither $A$ nor $B$ contains more than $2 n / 3$ vertices, and $C$ contains no more than $h^{3 / 2} n^{1 / 2}$ vertices. This extends a theorem of Lipton and Tarjan for planar graphs. We exhibit an algorithm which finds such a partition $(A, B, C)$ in time $O\left(h^{1 / 2} n^{1 / 2} m\right)$, where $m=|V(G)|+|E(G)|$.

Department of Mathematics, Sackler Faculty of Exact Science, Tel Aviv University, Ramat Aviv, Tel Aviv 69978, IsRael

Bellcore, 445 South Street, Morristown, New Jersey 07960

Dimacs Center, Hill Center, Busch Campus, Rutgers University, New Brunswick, NEW JERSEY 08903

School of Mathematics, Georgia Institute of Technology, Atlanta, Georgia 30332 